\title{
BER in slow-light and fast-light regimes of silicon photonic crystal waveguides: a comparative study
}

\author{
Jie You and Nicolae C. Panoiu
}

\begin{abstract}
In this paper, we present an in-depth comparison between the bit-error ratio (BER) of optical systems containing silicon photonic crystal (Si-PhC) waveguides (Si-PhCWs) operating in the slow- and fast-light regimes. Our analysis of these optical interconnects employs the time domain Karhunen-Loève expansion method for the statistical analysis of the optical signal and relies on a full theoretical model and its linearized form to describe the propagation of noisy optical signals in Si-PhCWs. These models incorporate all key linear and nonlinear optical effects and the mutual interaction between free-carriers and the optical field, as well as the influence of slow-light effects on the optical field and carriers dynamics. Using these tools and employing a 512-bit pseudorandom bit sequence, we have studied the dependence of BER on the key system parameters, including group-velocity, input power, and signal-to-noise ratio.
\end{abstract}

Index Terms-Optical interconnects, nonlinear optical signal processing, nonlinear optics, integrated optics.

\section{INTRODUCTION}

$\mathbf{T}$ HE drive towards exaflop supercomputers and the huge data transmission demands that are being created by large-scale cloud data centers are catalysts for increasing research efforts towards developing new system interconnects with highly improved performance [1], [2]. One promising alternative to the commonly used copper wires [3] are optical interconnects implemented in the silicon-on-insulator platform [4], [5], [6]. Importantly, due to the unique optical properties of silicon photonic waveguides (Si-PhWs) [7], [8], [9], employing silicon photonics based solutions for system interconnects does not simply amount to replacing copper wires with photonic wires, but they can also be used to implement key functionalities required by optical networks-on-chip. Thus, not only that Si-PhWs could facilitate ultrahigh bandwidth data communication [10], but also their strong dispersion and large optical nonlinearity allowed for the chip-level implementation of many functionalities, including optical modulators and switches [11], receivers [12], mode multiplexing [13], optical amplifiers [14], and frequency converters [15].

In addition to a constant drive towards downscaling the size of optical interconnects, an equally daunting challenge pertaining to exascale computing systems is to decrease the per-bit power consumption to levels that allow cost-effective operation. Photonic structures provide a versatile solution that addresses both these issues, as they allow one to engineer both the linear and nonlinear properties of the optical interconnects. To be more specific, by employing suitably engineered silicon photonic crystal $(\mathrm{Si}-\mathrm{PhC})$ waveguides $(\mathrm{Si}-\mathrm{PhCWs})$, one can

Jie You and Nicolae C. Panoiu are with the Department of Electronic and Electrical Engineering, University College London, Torrington Place, London WC1E 7JE, United Kingdom

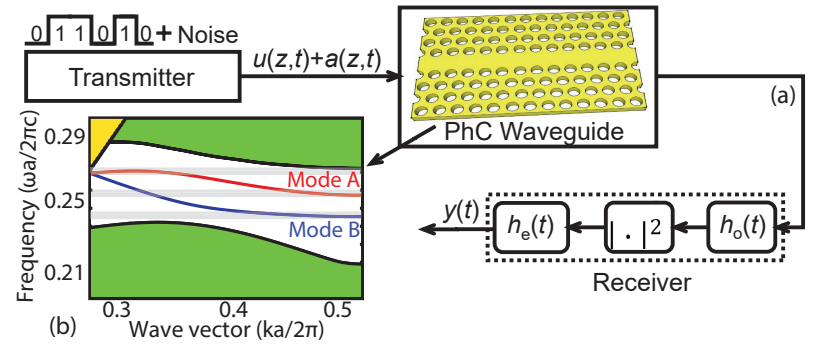

Fig. 1. (a) Schematic of the photonic system, containing a Si-PhCW and a direct-detection receiver composed of an optical filter, $h_{o}(t)$, a photodetector, and an electrical filter, $h_{e}(t)$. (b) Mode dispersion diagram of the Si-PhCW, with grey bands indicating the SL spectral domains $n_{g}>20$.

design optical interconnects that possess slow-light (SL) [16], [17] spectral regions characterized by significantly reduced group-velocity $(\mathrm{GV}), v_{g}$. This results in increased waveguide dispersion and effective waveguide nonlinearity, as these physical quantities scale with $v_{g}$ as $v_{g}^{-1}$ and $v_{g}^{-2}$, respectively. This means that when the optical interconnects are operated in the SL regime both the waveguide footprint and the optical power can be considerably reduced. A key issue in this context, which has yet to be investigated, is the extent to which the biterror ratio (BER) is affected when the waveguide operation is shifted from fast-light (FL) to the SL regime. This question is particularly important in the case of silicon waveguides because, unlike the thoroughly studied case of optical fibers, the optical field propagating in a silicon waveguide generates free-carriers (FCs), both this process and the $\mathrm{FC}$ dynamics being strongly dependent on the GV.

In this paper, we present a detailed analysis of the BER in Si-PhCWs, highlighting the key differences between the dependence of the BER on the parameters defining the optical signal and waveguide when the signal propagates in the FL or SL regime. With emphasis on the characteristics of BER in silicon optical interconnects, we consider a photonic system containing only a $\mathrm{Si}-\mathrm{PhCW}$, whose input and output are connected, respectively, to a transmitter and a directdetection optical receiver, shown in Fig. 1(a). However, our analysis can be easily extended to other components of optical networks, i.e. ring resonators, beam splitters, and multiplexers. An ON-OFF keying (OOK) modulated nonreturn-to-zero (NRZ) optical signal is inserted at the front-end of the system, with $\mathrm{ON}$ and $\mathrm{OFF}$ power values of $P=P_{0}$ and $P=0$, respectively, together with a stationary additive white Gaussian noise. Thus, we use a pseudorandom bit sequence (PRBS) of $2^{9}-1$ bits plus a zero bit, hence including all possible 9bit sequence patterns. In order to characterize the statistical properties of the transmitted signal we employ the time domain 
Karhunen-Loève (KL) expansion method [18], [19], [20], whereas the coupled dynamics of the optical field and FCs are described by a rigorous theoretical model that incorporates the linear and nonlinear optical effects pertaining to optical signal propagation in Si-PhCWs [21]. Importantly, the KL expansion method allows one to use much shorter PRBSs: whereas it already converges for PRBS-9, Monte-Carlo type methods could require PRBSs as long as $2^{32}$ to reach convergence.

\section{Silicon WaVeguide Structure And Properties}

The PhC slab waveguide considered here consists of a line defect created by filling in a line of holes oriented along the $\Gamma K$ direction of a hexagonal hole lattice in a silicon slab [see Fig. 1(a)]. The PhC has the lattice constant, $a$, hole radius, $r=0.22 a$, and slab thickness, $h=0.6 a$. The photonic band structure of the Si-PhCW, shown in Fig. 1(b), shows that the waveguide has two guiding modes, which are SL modes within certain spectral domains shown as grey bands in Fig. 1(b). In particular, the mode $A$ has two SL spectral domains, whereas mode $B$ only has one. In these SL regions the group index, $n_{g}=c / v_{g}$, second-order dispersion coefficient, $\beta_{2}=d^{2} \beta / d \omega^{2}$, where $\beta$ is the mode propagation constant, and nonlinear coefficient, $\gamma=3 \epsilon_{0} a \omega_{c} \Gamma /\left(4 v_{g} W\right)^{2}$ [22], with $\omega_{c}, \Gamma$, and $W$ being the carrier frequency of the signal, effective waveguide nonlinear susceptibility, and mode energy in the unit cell, respectively, have very large absolute values (see Fig. 2). This indicates that in the SL regime the linear and nonlinear optical effects are strongly enhanced.

\section{Optical Signal Propagation Model}

The dynamics of the optical field is described by a modified nonlinear Schrödinger equation, coupled to a rate equation for the FCs [21], [22]:

$$
\begin{aligned}
& j \frac{\partial u}{\partial z}-\frac{\beta_{2}}{2} \frac{\partial^{2} u}{\partial t^{2}}+\frac{j c \kappa}{2 n v_{g}} \alpha u+\frac{\omega_{c} \kappa}{n v_{g}} \delta n_{\mathrm{fc}} u+\gamma|u|^{2} u=0, \\
& \frac{\partial N}{\partial t}=-\frac{N}{t_{c}}+\frac{\gamma^{\prime \prime}}{\hbar \omega_{c} A}|u|^{4} .
\end{aligned}
$$

Here, $u(z, t)$ is the pulse envelope, measured in $\sqrt{\mathrm{W}}$, with $z$ and $t$ the distance and time along the $\mathrm{Si}-\mathrm{PhCW}$, respectively. The optical losses are quantified by $\alpha=\alpha_{\mathrm{in}}+\alpha_{\mathrm{fc}}$, with $\alpha_{\text {in }}$ being the intrinsic loss coefficient (in this work we set $\alpha_{\text {in }}=50 \mathrm{~dB} \mathrm{~cm}^{-1}$ ) and $\alpha_{\mathrm{fc}}=\sigma_{\alpha} N$ the FC absorption (FCA) coefficient, where $\bar{\lambda}=1550 \mathrm{~nm}, N$ is the FC density, and $\sigma_{\alpha}=1.45 \times 10^{-21}(\lambda / \bar{\lambda})^{2}$ (in units of $\mathrm{m}^{2}$ ). Moreover, $\delta n_{\mathrm{fc}}=\sigma_{n} N$ is the FC-induced refractive index change, where $\sigma_{n}=\sigma_{0}(\lambda / \bar{\lambda})^{2}$ (in units of $\mathrm{m}^{3}$ ), with $\sigma_{0}$ being a power dependent coefficient [7], $t_{c}$ is the FC relaxation time ( $t_{c}=0.5 \mathrm{~ns}$ in our analysis), $A$ is the averaged effective crosssectional area of the mode, and $\kappa$ the averaged overlap between the optical mode and the active waveguide area [22].

The noisy signal is expressed as the superposition of the optical CW signal with power $P(z)$ and a complex additive Gaussian noise, $a(z, t)$ :

$$
u(z, t)=[\sqrt{P(z)}+a(z, t)] e^{-j \Phi(z)},
$$
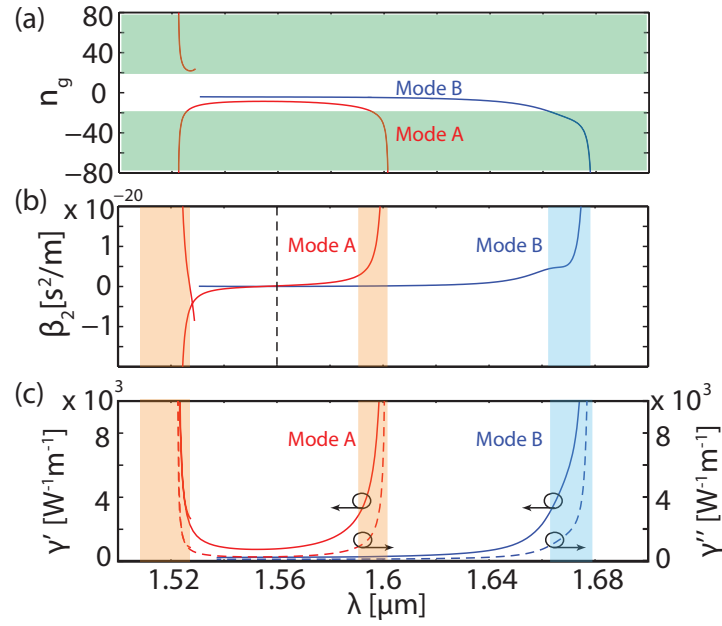

Fig. 2. (a), (b), (c) Wavelength dependence of $n_{g}, \beta_{2}$, and $\gamma^{\prime}$ and $\gamma^{\prime \prime}$, respectively, determined for mode $A$ (red lines) and mode $B$ (blue lines). The shaded areas correspond to the SL regime, defined by the relation $n_{g}>20$.

where $\Phi(z)$ is a global phase. In the steady-state regime $\frac{\partial N}{\partial t}=0$, which means that the steady-state FC density, $N_{s}$, is given by $N_{s}(z)=t_{c} \gamma^{\prime \prime} P^{2}(z) /\left(\hbar \omega_{c} A\right) \equiv \xi P^{2}(z)$.

We have used two methods to determine the optical field at the output of the Si-PhCW. In the first approach we solved numerically Eqs. (1), whereas in an alternative method we solved the following system of equations obtained by linearizing Eqs. (1) with respect to the noise $a(z, t)$ :

$$
\begin{aligned}
\frac{d P}{d z}= & -\frac{c \kappa}{n v_{g}}\left(\alpha_{\mathrm{in}}+\sigma_{\alpha} \xi P^{2}\right) P-2 \gamma^{\prime \prime} P^{2}, \\
\frac{d \Phi}{d z}= & -\frac{\omega_{c} \kappa}{n v_{g}} \sigma_{n} \xi P^{2}-\gamma^{\prime} P, \\
\frac{\partial a}{\partial z}= & -j \frac{\beta_{2}}{2} \frac{\partial^{2} a}{\partial t^{2}}-\frac{c \kappa}{2 n v_{g}} \alpha_{\mathrm{in}} a+j \gamma P\left(a+a^{*}\right)-\gamma^{\prime \prime} P a \\
& -\frac{c \kappa}{2 n v_{g}} \sigma_{\alpha} \xi P^{2}\left(3 a+2 a^{*}\right)+j \frac{2 \omega_{c} \kappa}{n v_{g}} \sigma_{n} \xi P^{2}\left(a+a^{*}\right),
\end{aligned}
$$

where the symbol "** indicates complex conjugation. These equations show that the power is independent on the phase and noise amplitude, its decay being due to intrinsic losses, FCA, and two-photon absorption (TPA). In addition, the variation of the total phase of the optical field is determined by the FC dispersion (FCD) and nonlinearly induced phase shift. Note also that due to the SL effects $\left(\gamma \sim v_{g}^{-2}\right)$, both $P$ and $\Phi$ vary much stronger with $z$ in the SL regime.

The key differences between the characteristics of the propagation of the optical signal in the FL and SL regimes are illustrated by Fig. 3. Thus, we have determined the time and wavelength domain evolution of a noisy signal in a $500 \mu \mathrm{m}$-long Si-PhCW, both in the FL $\left(n_{g}=10.3\right)$ and SL $\left(n_{g}=20.2\right)$ regimes, the bit sequence being "01101100". For completeness, we also show in Figs. 3(h) and 3(i) the dynamics of the photogenerated FCs. It can be seen that despite the fact that the bit sequence is preserved upon propagation in both cases, the optical signal and noise are distorted much more in the SL regime. In particular, the in-phase noise is strongly 

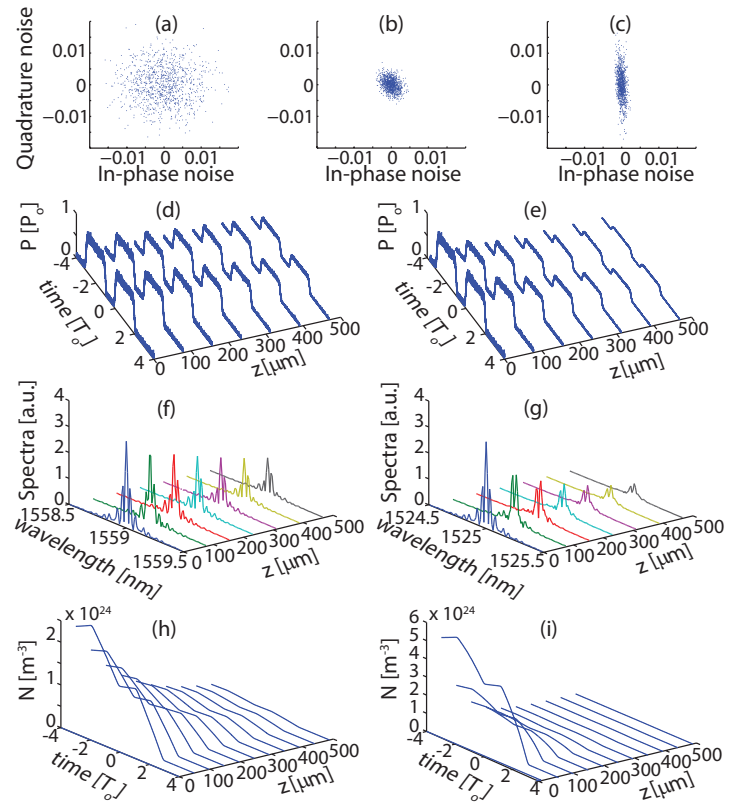

Fig. 3. (a) In-phase and quadrature noise components at the input of the SiPhCW. (b), (c) the same as in (a), but determined at the waveguide output in the FL and SL regimes. Second, third, and fourth row of panels show the time domain, spectral domain, and carrier density evolution of a noisy signal with $P_{0}=10 \mathrm{~mW}$ and $T_{0}=100 \mathrm{ps}$ in a $500 \mu \mathrm{m}$-long Si-PhCW, respectively, with the left (right) panels corresponding to the FL (SL) regime.

compressed in the SL regime, whereas the increased influence of intrinsic losses, FCA, and TPA on the optical field leads to much more rapid decay of the optical signal in the SL regime. As a result of enhanced linear and nonlinear optical effects, more than a double amount of FCs is generated in the SL regime, as per Figs. 3(h) and 3(i). As we will show in what follows, these qualitative differences in the dynamics of the optical signal have direct implications on the BER.

\section{RESUlTS AND Discussion}

In order to compare the system transmission BER in SL and FL regimes we assumed a noisy signal propagating in mode $A$, the carrier frequency, $\omega_{c}$, being chosen in such a way that the group index varied from $n_{g}=8.64$ in the FL regime to $n_{g}=34.3$ in SL case. The signal is assumed to be OOK modulated, with NRZ pulses in a back-to-back configuration and bit window of $T_{0}=100 \mathrm{ps}$ throughout our investigations. To calculate the transmission BER, we first propagated the optical signal in the $\mathrm{Si}-\mathrm{PhCW}$ using the linearized model, then determined the signal at the back-end of the directdetection receiver, and finally used the time-domain KL series expansion method to evaluate the BER [19], [20]. Importantly, the linearized model is accurate for the task at hand, and much less computationally demanding as compared to the full model. To model the direct-detection receiver, we assumed that the electrical filter is a low-pass integrate-and-dump filter with the $3-\mathrm{dB}$ bandwidth equal to $B_{e}=10 \mathrm{Gbs}^{-1}$, whereas the optical filter is a bandpass Lorentzian with 3-dB bandwidth, $B_{o}=4 B_{e}$. Specifically, the two filters are described by the following transfer functions, with $\Gamma_{o}=B_{o} / 2$ :
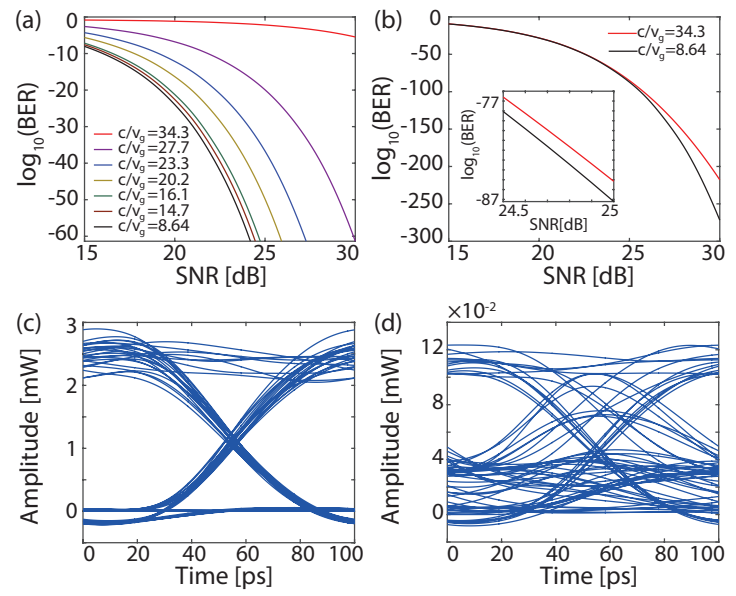

Fig. 4. Top panels show the system BER calculated for the Si-PhCW with FC dynamics included (left) and by neglecting them (right). Bottom panels show the eye diagrams corresponding to $n_{g}=8.64$ (left) and $n_{g}=27.7$ (right), both at $\mathrm{SNR}=25 \mathrm{~dB}$. In all panels, $P_{0}=10 \mathrm{~mW}$ and $L=500 \mu \mathrm{m}$.

$$
\begin{aligned}
& H_{o}^{i}(f)=\frac{\Gamma_{o}^{2}}{f^{2}+\Gamma_{o}^{2}}, \quad H_{o}^{q}(f)=-\frac{\Gamma_{o} f}{f^{2}+\Gamma_{o}^{2}}, \\
& H_{e}^{i}(f)=\left\{\begin{array}{cc}
1, & |f| \leq B_{e} / 2 \\
0, & |f|>B_{e} / 2
\end{array} \quad H_{e}^{q}(f)=0 .\right.
\end{aligned}
$$

The main parameter that determines the transmission BER is the $\mathrm{GV}$, as both the linear and nonlinear optical effects affecting the optical signal propagation strongly depend on it. In particular, by simply varying the frequency of the signal one can tune $v_{g}$ so as the optical signal propagation changes from the FL to the SL regime. To illustrate this, we varied the signal frequency while keeping constant the input power, $P_{0}=10 \mathrm{~mW}$, and waveguide length, $L=500 \mu \mathrm{m}$, and determined the dependence of BER on the signal-to-noise ratio (SNR). The outcomes of this analysis, summarized in Fig. 4(a), show that as the signal is tuned deeper into the SL regime by changing $n_{g}$ from 8.64 to 34.3 the signal impairments increase dramatically, the BER varying by $60 \mathrm{~dB}$ when $\mathrm{SNR}=25 \mathrm{~dB}$.

These results raise a key question: is this signal degradation primarily related to the linear and nonlinear optical effects in the $\mathrm{Si}-\mathrm{PhCW}$ or it is due the influence of the generated FCs on the signal propagation? To answer this question, we investigated two cases of optical signal propagation in the Si$\mathrm{PhCW}$, in both instances setting the FC density to zero by imposing $\gamma^{\prime \prime}=0$. For a better illustration of the conclusion of this study, we performed the simulations for the largest and smallest value of $n_{g}$ in Fig. 4(a), the results being shown in Fig. 4(b). Thus, it can be seen that the variation of BER with SNR only weakly depends on $n_{g}$, which means that, unlike optical fibers, the BER is primarily determined by the FCs. Also, our calculations show that in the presence of FCs the FCA characteristic length is smaller than that of TPA, for all values of $n_{g}$, which proves that FCA effects are stronger. In addition, the system performance without FCs is far better than in the case when FCs dynamics are included, especially in the SL regime. The degradation of the transmitted signal when the $\mathrm{Si}-\mathrm{PhCW}$ operation is shifted from the FL to the SL regime is illustrated by the eye diagrams presented in Figs. 4(c) and 4(d), 

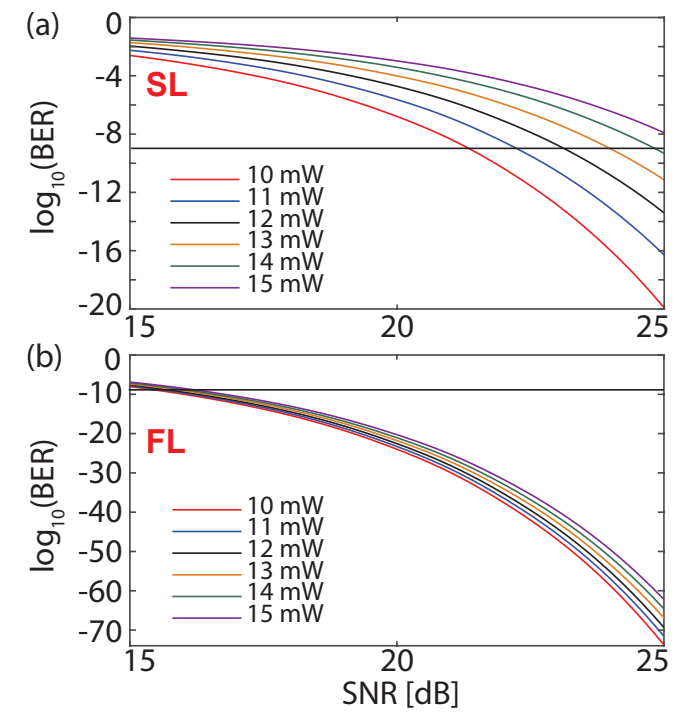

Fig. 5. (a), (b) System BER vs. SNR, calculated for different $P_{0}$, in the SL $\left(n_{g}=27.7\right)$ and FL $\left(n_{g}=8.64\right)$ regimes, respectively. The horizontal black line indicates a BER of $10^{-9}$.

too. Due to increased optical interactions in the SL regime, the eye diagram almost completely closes as $n_{g}$ increases from 8.64 to 27.7 , with strong power fluctuations and bit window shifts being observed in the SL regime.

Since the optical power is the main parameter that determines the strength of the nonlinear optical effects, including the TPA as the source of FCs, we have calculated the dependence of the BER on the SNR, for different values of $P_{0}$. The results of these calculations, presented in Fig. 5, show that, irrespective of $P_{0}$, a much better system performance is achieved in the FL regime $\left(n_{g}=8.64\right)$ than in the SL regime $\left(n_{g}=27.7\right)$. This is primarily due to the fact that the $\mathrm{Si}$ PhCW operating in the SL regime has a much larger nonlinear coefficient, which leads both to a larger parametric gain amplification of the noise and to increased amount of FCs. To be more specific, $\gamma$ is proportional to $v_{g}^{-2}$ and therefore in the SL regime the parametric gain responsible for noise amplification $\left(\sim \gamma^{\prime}\right)$ and TPA $\left(\sim \gamma^{\prime \prime}\right)$ are enhanced. Consequently, the signal degradation increases, leading to larger BER. In addition, TPA increases with $P_{0}$, too, which explains why the BER increases with $P_{0}$. One last idea illustrated by Fig. 5 is that in the SL regime the BER varies much stronger with $P_{0}$ as compared to this power variation in the FL regime.

\section{CONCLUSION}

In conclusion, we studied the transmission BER in silicon photonic crystal waveguides and contrasted the results obtained in two relevant cases, namely when the optical signal propagates in the fast- and slow-light regimes. Our analysis revealed that although slow-light effects provide the key advantage of increased nonlinearity, they also lead to detrimental consequences, including a significant degradation of the transmission BER. Theoretical and computational investigations showed that the signal impairments are primarily due to the generation of free carriers.

\section{REFERENCES}

[1] D. A. B. Miller, "Rationale and challenges for optical interconnects to electronic chips," Proc. IEEE, vol. 88, no. 6, pp. 728-749, Jun. 2000.

[2] J. A. Kash, A. F. Benner, F. E. Doany, D. M. Kuchta, B. G. Lee, P. K. Pepeljugoski, L. Schares, C. L. Schow, and M. Taubenblatt,“Optical Interconnects in Exascale Supercomputers," $23^{\text {rd }}$ Annual Meeting of the IEEE Photonics Society, Nov. 2010, pp. 483-484.

[3] R. Ho, K. W. Mai, and M. A. Horowitz, "The future of wires," Proc. IEEE, vol. 89, no. 4 , pp. 490-504, Apr. 2001

[4] K. K. Lee, D. R. Lim, H. C. Luan, A. Agarwal, J. Foresi, and L. C. Kimerling,"Effect of size and roughness on light transmission in a $\mathrm{Si} / \mathrm{SiO}_{2}$ waveguide: Experiments and model," Appl. Phys. Lett., vol. 77. no. 11, pp. 1617-1619, Sep. 2000.

[5] R. U. Ahmad, F. Pizzuto, G. S. Camarda, R. L. Espinola, H. Rao, and R. M. Osgood,"Ultracompact corner-mirrors and T-branches in siliconon-insulator," IEEE Photon. Technol. Lett., vol. 14, no. 1, pp. 65-67, Jan. 2002.

[6] S. Rumley, D. Nikolova, R. Hendry, Q. Li, D. Calhoun, and K. Bergman, "Silicon Photonics for Exascale Systems," J. Lightw. Technol., vol. 33 no. 3, pp. 547-562, Feb. 2015.

[7] Q. Lin, O. J. Painter, and G. P. Agrawal,"Nonlinear optical phenomena in silicon waveguides: Modeling and applications," Opt. Express, vol. 15, no. 25, pp. 16604-16644, Nov. 2007.

[8] R. M. Osgood, N. C. Panoiu, J. I. Dadap, X. Liu, X. Chen, I-W. Hsieh, E. Dulkeith, W. M. J. Green, and Y. A. Vlassov,"Engineering nonlinearities in nanoscale optical systems: physics and applications in dispersionengineered silicon nanophotonic wires," Adv. Opt. Photon., vol. 1, no. 1, pp. 162-235, Nov. 2009.

[9] J. B. Driscoll, R. M. Osgood, R. R. Grote, J. I. Dadap, and N. C. Panoiu, "Squeezing Light in Wires: Fundamental Optical Properties of Si Nanowire Waveguides," J. Lightw. Technol., vol. 33, no. 14, pp. 31163131, Jul. 2015.

[10] B. G. Lee, X. Chen, A. Biberman, X. Liu, I-W. Hsieh, C. Chou, J. I. Dadap, F. Xia, W. M. J. Green, L. Sekaric, Y. A. Vlasov, R. M. Osgood, and K. Bergman, "Ultrahigh-Bandwidth Silicon Photonic Nanowire Waveguides for On-Chip Networks," IEEE Photon. Technol. Lett., vol. 20, no. 5-8, pp. 398-400, Mar.-Apr. 2008.

[11] B. G. Lee, A. Biberman, P. Dong, M. Lipson, and K. Bergman, "Alloptical comb switch for multiwavelength message routing in silicon photonic networks," IEEE Photon. Technol. Lett., vol. 20, no. 9-12, pp. 767-769, May.-Jun. 2008.

[12] S. Assefa, F. Xia, W. M. J. Green, C. L. Schow, A. V. Rylyakov, and Y. A. Vlasov, "CMOS-Integrated Optical Receivers for On-Chip Interconnects," IEEE J. Sel. Top. Quantum Electron., vol. 16, no. 5, pp. 1376-1385, Sep.-Oct. 2010.

[13] B. Stern, X. Zhu, C. P. Chen, L. D. Tzuang, J. Cardenas, K. Bergman, and M. Lipson, "On-chip mode-division multiplexing switch," Optica, vol. 2, no. 6, pp. 530-535, Jun. 2015.

[14] R. Espinola, J. I. Dadap, R. M. Osgood, S. J. McNab, and Y. A. Vlasov, "Raman amplification in ultrasmall silicon-on-insulator wire waveguides," Opt. Express, vol. 12, no. 16, pp. 3713-3718, Aug. 2004.

[15] S. Zlatanovic, J. S. Park, S. Moro, J. M. C. Boggio, I. B. Divliansky, N. Alic, S. Mookherjea, and S. Radic, "Mid-infrared wavelength conversion in silicon waveguides using ultracompact telecom-band-derived pump source," Nat. Photonics, vol. 4, no. 8, pp. 561-564, Aug. 2010.

[16] T. F. Krauss, "Why do we need slow light?," Nat. Photonics, vol. 2, no. 8, pp. 448-450, Aug. 2008.

[17] T. Baba, "Slow light in photonic crystals," Nat. Photonics, vol. 2, no. 8, pp. 465-473, Aug. 2008.

[18] A. Papoulis, Probability, Random Variables, and Stochastic Processes, 3rd ed. New York, NY, USA: McGraw-Hill, 1991, p.329.

[19] G. Bosco, A. Carena, V. Curri, R. Gaudino, P. Poggiolini, and S. Benedetto, "A novel analytical approach to the evaluation of the impact of fiber parametric gain on the bit error rate," IEEE Trans. Commun., vol. 49, no. 12, pp. 2154-2163, Dec. 2001.

[20] J. You and N. C. Panoiu,"Calculation of Bit Error Rates in Optical Systems with Silicon Photonic Wires," IEEE J. Quantum Electron., vol. 51, no. 4, art.no.8400108, Apr. 2015.

[21] N. C. Panoiu, J. F. McMillan, and C. W. Wong, "Theoretical Analysis of Pulse Dynamics in Silicon Photonic Crystal Wire Waveguides," IEEE J. Sel. Top. Quantum Electron., vol. 16, no. 1, pp. 257-266, Jan-Feb. 2010.

[22] S. Lavdas and N. C. Panoiu, "Theory of pulsed four-wave mixing in one-dimensional silicon photonic crystal slab waveguides," Phys. Rev. B , vol. 93, no. 11, art. no. 115435, Mar. 2016. 